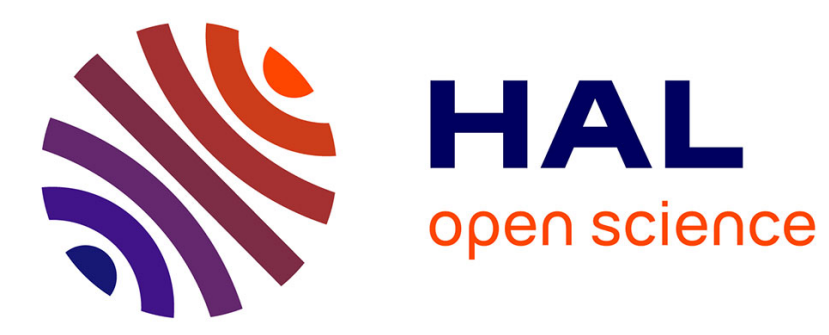

\title{
Une approche du séchage de matériaux fortement déformables
}

\author{
W. Aregba, W. Jomaa, J.R. Puiggali
}

\section{To cite this version:}

W. Aregba, W. Jomaa, J.R. Puiggali. Une approche du séchage de matériaux fortement déformables. Revue de Physique Appliquée, 1990, 25 (11), pp.1161-1175. 10.1051/rphysap:0199000250110116100 . jpa-00246285

\section{HAL Id: jpa-00246285 https://hal.science/jpa-00246285}

Submitted on 1 Jan 1990

HAL is a multi-disciplinary open access archive for the deposit and dissemination of scientific research documents, whether they are published or not. The documents may come from teaching and research institutions in France or abroad, or from public or private research centers.
L'archive ouverte pluridisciplinaire HAL, est destinée au dépôt et à la diffusion de documents scientifiques de niveau recherche, publiés ou non, émanant des établissements d'enseignement et de recherche français ou étrangers, des laboratoires publics ou privés. 
Classification

Physics Abstracts

$05.60-66.20-46.20$

\title{
Une approche du séchage de matériaux fortement déformables
}

\author{
W. Aregba, W. Jomaa et J. R. Puiggali \\ Laboratoire Energétique et Phénomènes de Transfert, L.E.P.T. - E.N.S.A.M., associé au C.N.R.S., \\ Esplanade des Arts et Métiers, 33405 Talence Cedex, France
}

(Reçu le 20 février 1990, révisé le 25 avril 1990, accepté le 10 juillet 1990)

\begin{abstract}
Résumé. - Le séchage de matériaux déformables est abordé au niveau expérimental. Puis un modèle de séchage diffusif est construit, qui rend compte des spécificités des matériaux grâce à un terme de transport convectif lié au retrait. Une étude de sensibilité sur ce terme convectif est ensuite menée. Cette étude permet de préciser sous quelles conditions à la fois de séchage et de matériau, le terme convectif est nécessaire pour prendre en compte, dans un problème de séchage, le caractère déformable.

Abstract. - In an experimenal way, the drying of shrinking materials was studied. Then, a diffusive drying model was built which account for the specificities of such materials, with the help of a convective transport term connected to the shrinkage. A sensitivity study was led on that convective term. This study allowed us to specify the conditions (drying and material) under which the convective term cannot be neglected.
\end{abstract}

\section{Introduction.}

L'opération de séchage constitue un maillon incontournable dans de nombreux procédés d'élaboration qui conduisent des matériaux de base au produit fini. Aussi les matériaux à sécher sont divers et variés tant dans leur nature physico-chimique que dans leur forme et leur comportement au cours de séchage [1].

L'opération de séchage doit respecter certains critères de qualité liés au produit tout en garantissant, pour la chaîne de production une cadence et un coût $[2,3]$.

Ces considérations ont conduit au développement de nombreux travaux aux diverses échelles classiques du raisonnement: le séchoir, le produit et les processus internes.

$\mathrm{u}$ ar icu-

lièrement à la modélisation des processus internes de transfert de matière et de chaleur qui contribuent au séchage de matériaux qui se déforment fortement au cours de l'opération.

Dans la littérature les références sur la modélisation des transports internes contribuant au séchage de matériaux, à squelette rigide, sont nombreuses et nous n'en citerons que quelques unes [4-12]. Par contre, il n'en est pas de même pour ce qui est spécifiquement du séchage des milieux fortement déformables [13-18].
Même si ces matériaux sont communément utilisés dans l'industrie, l'étude fine de leur comportement au séchage pose des problèmes aussi bien d'ordre technique que théorique :

- du point de vue technique la difficulté sera la définition d'une préforme en vue de l'obtention, en fin de séchage, d'une forme finale prédéfinie, nous ne développerons pas ici cette problématique,

- du point de vue théorique, avant de définir un modèle de connaissance susceptible de décrire de manière correcte les mécanismes qui entrent en jeu lors du séchage des matériaux déformables, il nous semble nécessaire de répondre, comme le suggère Aregba [19], aux deux questions suivantes:

- la con $r$

séchage entraîne-t-elle forcément la compression ou la distorsion de son squelette solide?

- dans quelle mesure la contraction du matériau liée à la migration du solvant influe sur cette migration?

Nous analysons, dans ce texte, au regard des réponses à ces deux questions, l'évolution des modèles classiques de séchage, en vue de prendre en compte la spécificité des matériaux déformables.

Crapiste et al. [13], Rao et al. [14], Haghighi et al. [15], Sih et al. [16] ont proposé des modèles issus de 
la mécanique des milieux continus. Ces modèles sont basés sur les équations de bilans qui font intervenir les vitesses de déplacement des phases fluide et solide.

Pour déterminer la vitesse de déplacement de la phase solide, deux démarches différentes sont possibles :

- Rao et al. [14], Haghighi et al. [15], Sih et al. [16] introduisent les lois de comportement rhéologiques des différents matériaux;

- dans le cas d'un déplacement unidirectionnel Crapiste et al. [13] déterminent cette vitesse à partir de l'équation de continuité de la phase solide et de la fonction retrait déterminée expérimentalement; c'est dans le cadre général de cette démarche que nous faisons par la suite nos développements.

La modélisation du séchage des produits fortements déformables peut nécessiter la prise en compte de l'interaction entre mécanismes de transport du solvant et comportement mécanique de la matrice solide. Cette interaction peut se traduire par un terme lié au gradient de contrainte dans l'équation de transport. Le support expérimental de notre étude est constitué de deux matériaux : un Gel de PolyAcrilamide (G.P.A.) et une pâte de cellulose. Pour ces matériaux nous avons analysé l'évolution des grandeurs caractéristiques en terme de séchage et de retrait. Comme nous le montrons par la suite, cette analyse nous permet de dégager deux idées :

- la déformation du matériau ne dépend que de l'état hydrique de celui-ci (i.e. sa teneur en eau moyenne). L'interaction entre mécanismes de transport et comportement mécanique en terme de contraintes est alors peu significative,

- les effets des déformations induites par la perte en eau sur le transport du solvant ne sont pas négligeables.

Aussi, dans ce cas le séchage du milieu déformable peut être décrit par un modèle diffusif tenant compte de la déformation au travers d'un terme convectif et ayant une condition à la limite du type frontière libre.

Ce modèle est ensuite utilisé pour simuler un procédé de séchage convectif à basse et moyenne température. Les résultats numériques et expérimentaux sont comparés et commentés.

Une étude de sensibilité aux différents termes de l'équation en teneur en eau est effectuée pour préciser dans quelles situations (conditions de séchage et matériaux) le modèle diffusif classique (c'est-à-dire sans le terme convectif) est suffisant pour assurer une bonne description du séchage malgré le caractère déformable du matériau.

\section{Propriétés des matériaux.}

1.1 PréSentation SuCCinte des matériauX. Le G.P.A. est obtenu par copolymérisation radicalaire d'unités bifonctionnelles d'acrylamide et quadrifonctionnelles de biacrylamide. Le matériau ainsi obtenu est constitué d'un réseau de polymère et d'un fluide essentiellement de l'eau présente entre les mailles du réseau. Nous considérons le G.P.A. comme un milieu biphasique et microporeux [20], [21]. La teneur en eau initiale $\left(\langle W\rangle_{0}\right)$ du G.P.A. est voisine de 6 , base sèche, et correspond à l'état de saturation.

La pâte de cellulose est obtenue à partir de cellulose purifiée, la cellulose est restructurée à l'aide de procédés physico-chimiques tel le procédé viscose [22]. Le produit obtenu présente un degré de polymérisation élevé ce qui garantit les performances mécaniques du matériau. La structure de ce matériau est une cohabitation désordonnée de zones amorphes et zones cristallines. Nous considérons cette pâte comme isotrope, sa teneur en eau initiale $\left(\langle W\rangle_{0}\right)$, correspondant à l'état de saturation, est voisine de 5 , toujours base sèche.

\subsection{L'ÉQUilibre HYGROSCOPIQUE. - Comme} toute étude sur le séchage d'un matériau, l'étape incontournable consiste à évaluer son caractère hygroscopique, caractère qui indique l'affinité que peut avoir le matériau avec son milieu environnant.

La connaissance de la courbe de désorption permet d'identifier les types d'eau présents dans le matériau (eau libre dans les cavités, dont la quantité est limitée par la porosité ; eau liée adsorbée dans les parois du squelette solide), et donc d'avoir une première information sur les termes moteurs du transport et sur l'allure de la vitesse de séchage ou du flux-masse d'eau évaporée.

La limite entre l'eau libre et l'eau liée définit le point de saturation de la matrice solide. Le matériau rentre dans le domaine hygroscopique quand sa teneur en eau passe en dessous de ce point.

Sur les figures $1.1 \mathrm{a}$ et $1.1 \mathrm{~b}$ sont représentées les isothermes de désorption (teneur en eau versus l'humidité relative de l'ambiance) des deux matériaux, obtenues par la méthode des solutions salines saturées. On notera ici que les échantillons utilisés sont des couches minces pour qu'à l'état d'équilibre (surface-ambiance) il n'y est pas de gradients internes de teneur en eau.

Compte tenu des teneurs en eau initiales et des courbes de désorption, les matériaux commenceront à sécher dans le domaine non hygroscopique pour passer ensuite dans le domaine hygroscopique régi par l'isotherme de désorption.

Pour décrire ces isothermes, de nombreuses corrélations existent dans la littérature comme l'indiquent les revues de Bizot et al. [23] et Nelson [24]. Nous 

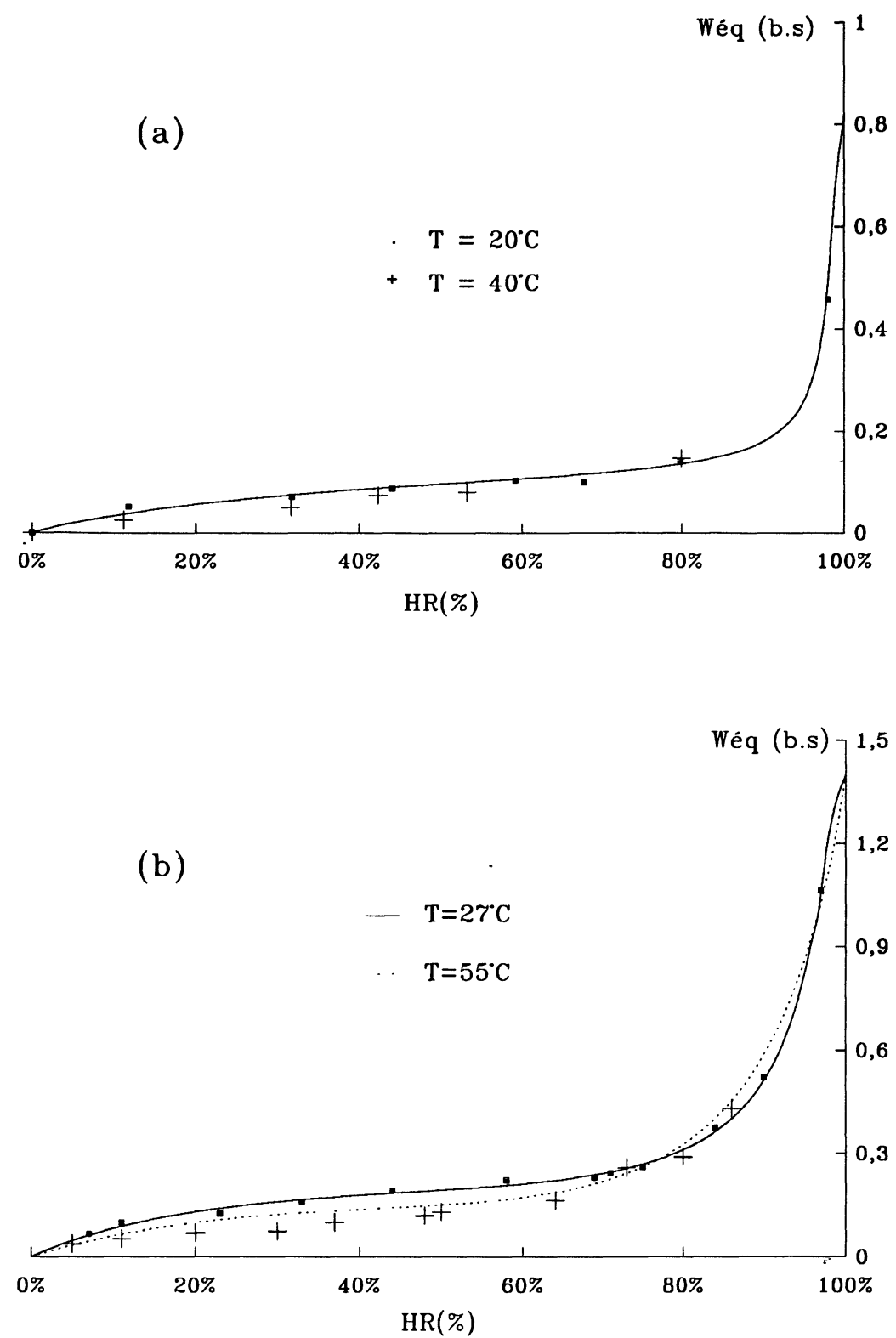

Fig. 1.1. - Isothermes de désorption, a) cellulose purifiée, b) G.P.A.

[Desorption isotherms : a) regenerated cellulose, b) polyacralamid gel (G.P.A.).]

avons pour notre part retenu celle de d'Arcy et Watt [25] qui est une fonction à 5 paramètres, sans pour autant attribuer de sens physique particulier aux coefficients identifiés dans la corrélation. De plus, dans la plage de température explorée expérimentalement, nous n'avons pas mis en évidence de dépendances significative des coefficients avec la température.

1.3 LA MASSE VOlumiQue ET LE RETRAIT. - La connaissance de l'évolution de la masse volumique et du retrait volumique du matériau au cours du séchage est essentielle et ceci pour deux raisons principales :

- la masse volumique intervient, entre autre, dans le calcul des coefficients de diffusion thermique et massique. La connaissance de son évolution en fonction de la teneur en eau est donc nécessaire our le calcul des termes moteurs des processus de séchage,

- nous verrons dans la suite en quoi la connaissance du retrait volumique et de la masse volumique peuvent aider à préciser la nature du matériau ainsi que son comportement au cours du séchage.

Le retrait volumique est défini comme suit :

$$
R_{\mathrm{v}}=\frac{V(\langle W\rangle)}{V_{0}}
$$

Dans cette relation $\langle W\rangle$ est la teneur en eau 
moyenne du matériau, $V(\langle W\rangle)$ son volume à cette teneur en eau moyenne et $V_{0}$ son volume initial.

De prime abord il est pensable que l'évolution de ces deux quantités dépende intrinsèquement des conditions de séchage, car la déformation du matériau peut dépendre des gradients de température et de teneur en eau au sein du matériau : ces gradients étant quant à eux différents pour deux conditions de séchage différentes. Les expériences effectuées sur nos produits montrent que la masse volumique ainsi que le retrait ne dépendent pas des conditions de séchage (les conditions explorées correspondent à un séchage convectif à basse et moyenne température, i.e. $30^{\circ} \mathrm{C} \leq T_{\infty} \leq 80^{\circ} \mathrm{C}$ ), mais ne dépendent que de la teneur en eau moyenne $(\langle W\rangle)$ à laquelle est le matériau (Figs. 1.2a et b). Nous pensons alors que la migration du solvant n'entraîne pas la distortion du squelette solide, mais plutôt un rapprochement des éléments de ce squelette. Ceci nous permet d'affirmer que le couplage entre les effets du séchage et les contraintes mécaniques se fait simplement à l'aide d'une loi de comportement du matériau qui relie le retrait volumique à la teneur en eau, cette idée sera précisée ultérieurement.
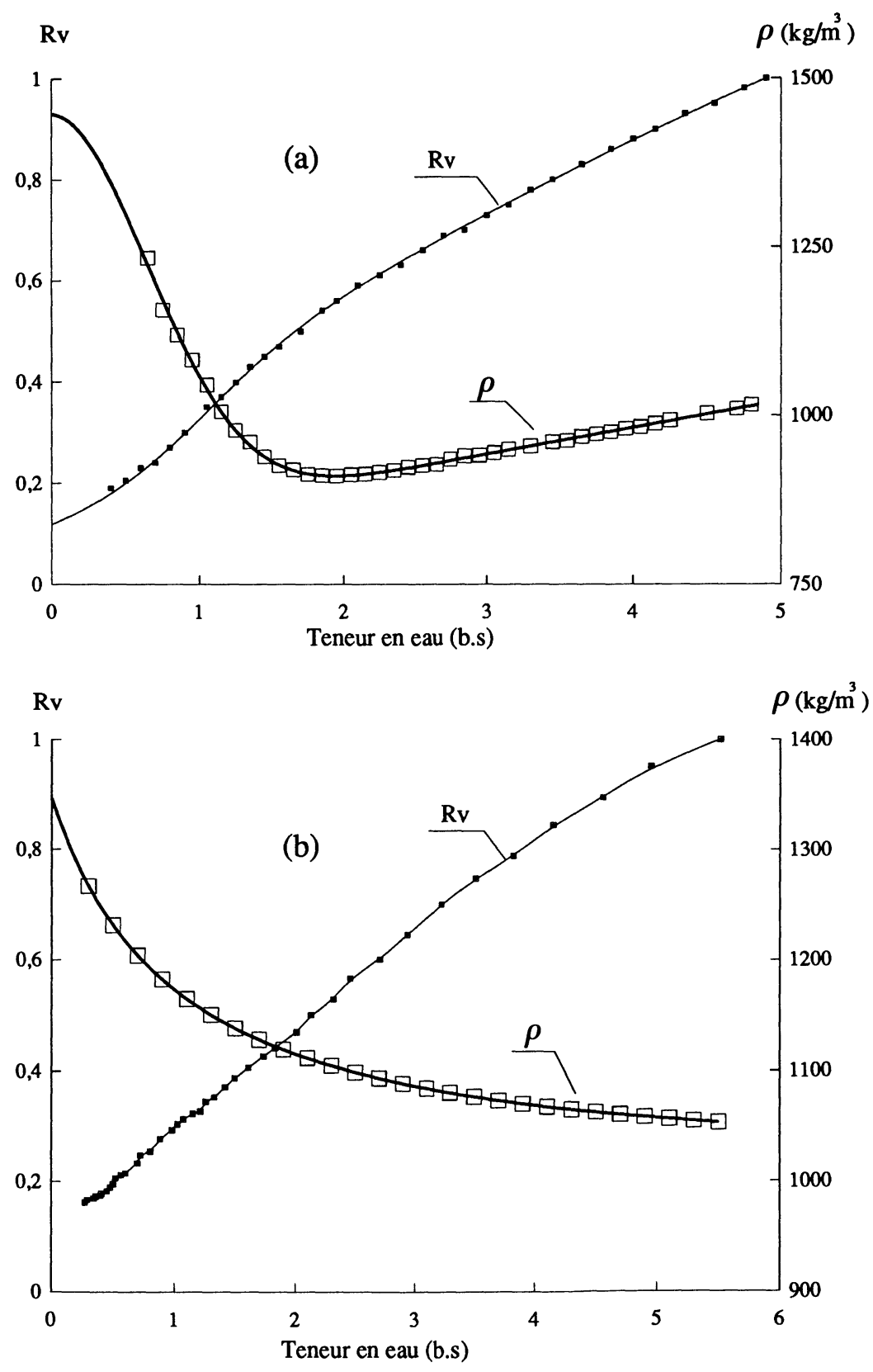

Fig. 1.2. - Evolution de la masse volumique et du retrait volumique : a) cellulose purifiée, b) G.P.A.

[Bulk density and volumic shrinkage : a) regenerated cellulose, b) polyacralamid gel (G.P.A.).] 


\section{Modélisation du séchage des matériaux.}

2.1 CARACTÉRISATION DU RETRAIT VOLUMiQue. - Dans le matériau les éléments de la matière solide sont séparés par des couches d'eau qui sont soit piégées dans les interstices de la matrice solide par capillarité, soit adsorbées au squelette. Lors de la migration de l'eau, liée à un processus de séchage par exemple, il y a resserrement des éléments de la matière solide, ce resserrement correspond au retrait, si en plus il y a distortion de la matière solide, on parle de déformation.

Le retrait idéal est la situation pendant laquelle on a proportionnalité entre la masse d'eau évaporée et la réduction du volume du matériau. De plus, le retrait peut être isotrope ou anisotrope. Enfin, sous des conditions de séchage non homogènes, le retrait peut être contrarié.

Les expériences, effectuées sur nos matériaux, montrent que leur retrait est indépendant des conditions de séchage, et que, pour des conditions de séchage homogènes (les mêmes sur toutes les faces qui participent alors aux échanges de chaleur et de masse) nous avons un retrait libre (non contrarié) avec conservation de la géométrie du matériau.

Lors du séchage nous avons, excepté pour les faibles teneurs en eau, proportionnalité entre la masse d'eau évaporée et la réduction du volume. Nous considérons alors que le matériau est en situation de retrait idéal (Figs. 1.2a et b).

Pour aider à l'analyse, nous introduisons le facteur de contraction $(\chi)$ qui donne une indication qualita-

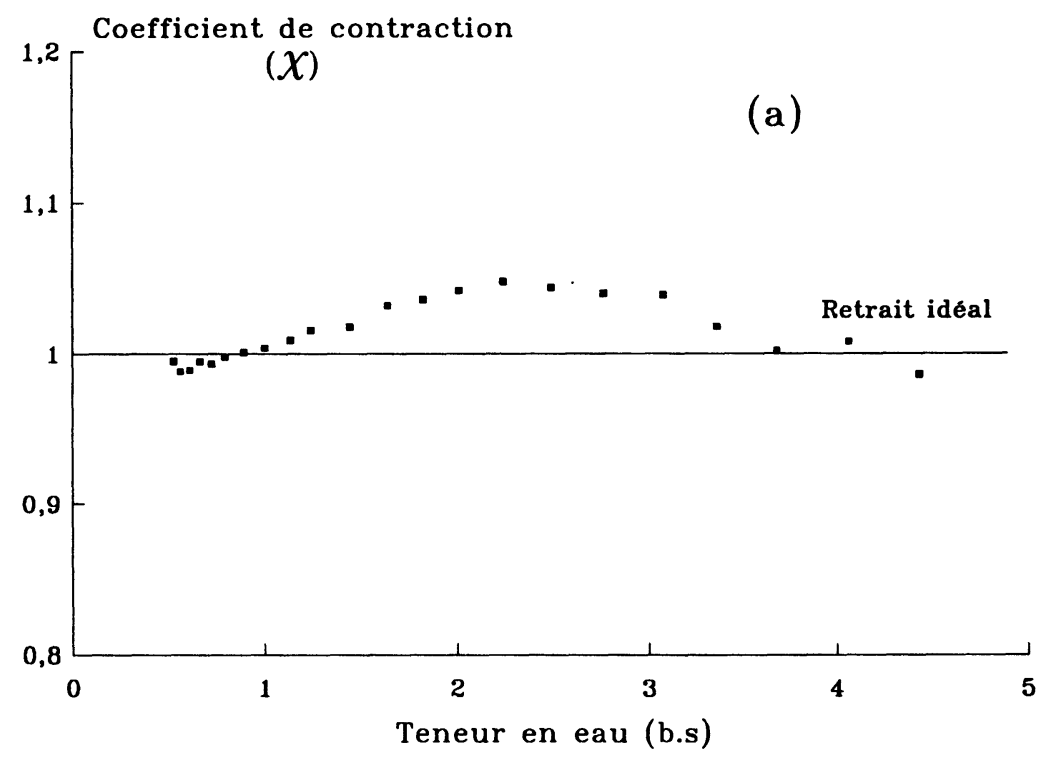

${ }_{1,2}^{\text {Coefficient de contraction }}$

(b)

1,1

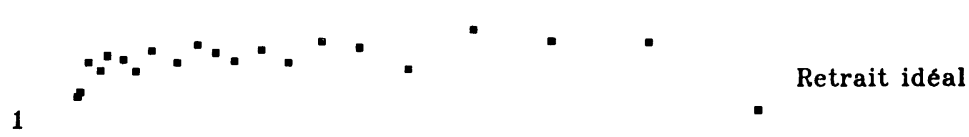

0,9

0,8

$$
\stackrel{2}{2}{ }^{3} \text { Teneur en eau (b.s) }
$$

Fig. 2.1. - Coefficient de contraction : a) cellulose purifiée, b) G.P.A.

[Contraction coefficient : a) regenerated cellulose, b) polyacralamid gel (G.P.A.).] 
tive sur la formation, ou non, de cavités lors du retrait. Ce facteur est défini comme suit:

$$
\chi=1+\frac{\Delta V_{\mathrm{c}}}{\Delta V(\langle W\rangle)}
$$

dans cette définition $V_{\mathrm{c}}$ est le volume des cavités à l'instant où la teneur en eau moyenne vaut $(\langle W\rangle)$. Ce facteur, $\chi$, provient d'une hypothèse d'additivité entre les volumes des phases solide, liquide et les volumes des cavités et de l'hypothèse de conservation du volume de la phase solide.

$$
\mathrm{d} V(\langle W\rangle)=\mathrm{d} V_{\mathrm{s}}+\mathrm{d} V_{1}+\mathrm{d} V_{\mathrm{c}}
$$

avec :

$$
\left.\begin{array}{c}
\mathrm{d} V=-\Delta V, \mathrm{~d} V_{1}=-\Delta V_{1}, \mathrm{~d} V_{\mathrm{c}}=\Delta V_{\mathrm{c}} \\
V(\langle W\rangle)=V_{\mathrm{s}}+V_{1}+V_{\mathrm{c}} \\
-\Delta V(\langle W\rangle)=-\Delta V_{1}+\Delta V_{\mathrm{c}}
\end{array}\right\} \Rightarrow \chi=\frac{\Delta V_{1}}{\Delta V(\langle W\rangle)}
$$

Kneule [26] classe les différents types de retraits en fonction du coefficient de contraction. Les évolutions du facteur de contraction en fonction de la teneur en eau moyenne (Figs. 2.1a et b) montrent que nous pouvons, en première approche, nous
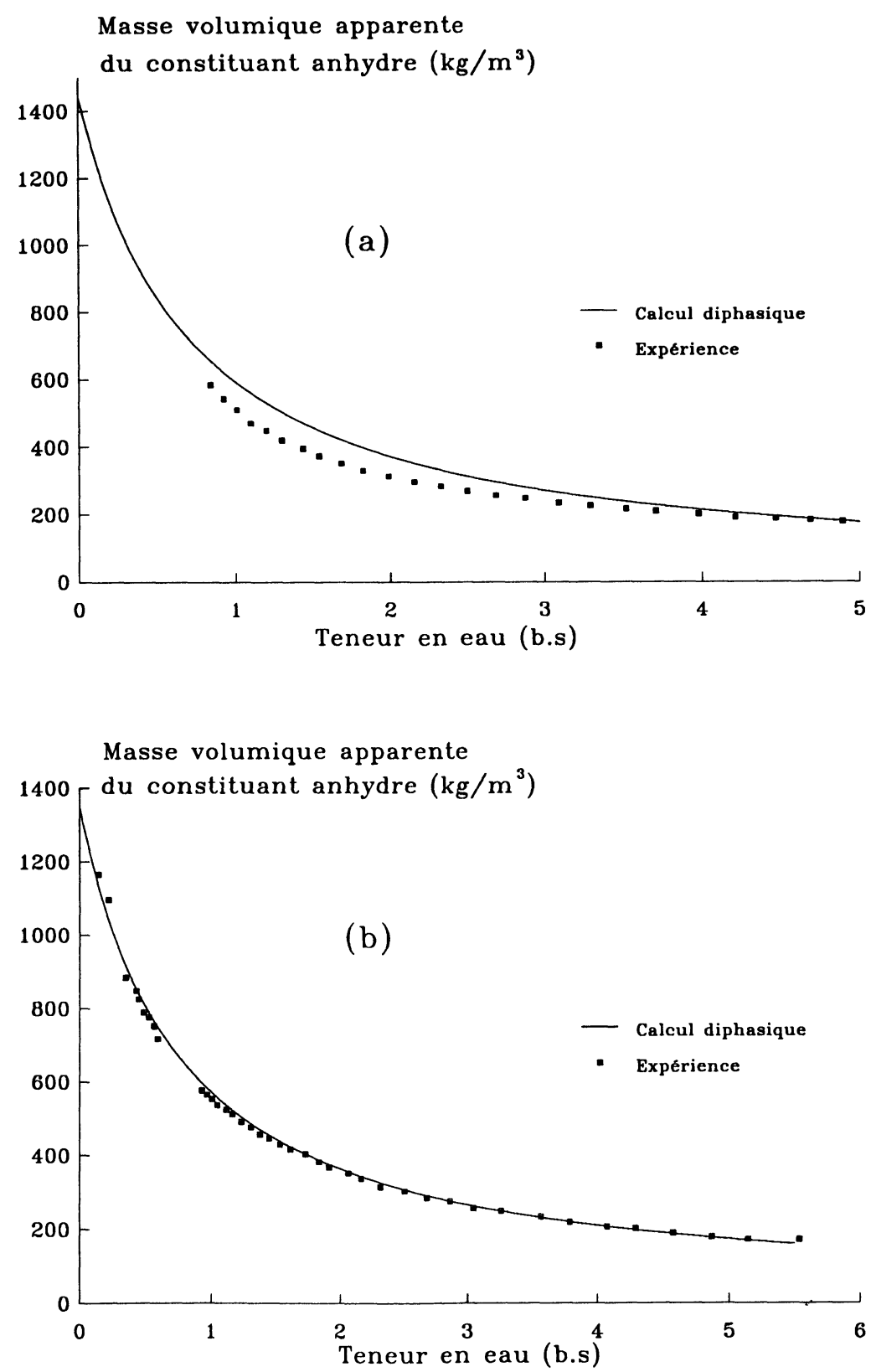

Fig. 2.2. - Evolution de la masse volumique apparente du constituant anhydre : a) cellulose purifiée, b) G.P.A.

[Apparent density of the solid phase : a) regenerated cellulose, b) polyacralamid gel (G.P.A.).] 
situer dans le cas où $\chi$ vaut 1 . Ceci revient à dire que le retrait est régulier et sans formation de cavités, limitant ainsi les effets de pression en phase gazeuse. Nous sommes alors dans une situation de retrait idéal.

Un autre paramètre utile est la masse volumique apparente de la matière solide [27] $\rho_{\mathrm{s}}^{\mathrm{a}}$ définie comme suit :

$$
\rho_{\mathrm{s}}^{\mathrm{a}}=\frac{m_{\mathrm{s}}}{V(\langle W\rangle)}
$$

Dans cette définition, $m_{\mathrm{s}}$ est la masse de la matière solide.

Dans l'hypothèse, d'un milieu diphasique la relation suivante exprime l'additivité des volumes de la matière solide et de l'eau :

$$
\rho_{\mathrm{s}}^{\mathrm{a}}=\frac{\rho_{\mathrm{s}} \cdot \rho_{1}}{\rho_{1}+\rho_{\mathrm{s}} \cdot(\langle W\rangle)}
$$

Dans cette relation $\rho_{\mathrm{s}}$ et $\rho_{1}$ sont les masses volumiques intrinsèques de la matière solide sèche et de la phase liquide (eau).

Sur les figures $2.2 \mathrm{a}$ et $2.2 \mathrm{~b}$ sont reportées les évolutions de la masse volumique apparente obtenue expérimentalement et celle obtenue en utilisant la relation précédente. La relative bonne concordance entre ces évolutions montre que les milieux peuvent être considérés comme diphasiques.

L'analyse des coefficients de contraction $(\chi)$ et du caractère diphasique des matériaux, nous permet de décrire le séchage de tels matériaux par un modèle de transport liquide diffusif.

2.2 Mise EN PLACE DU MODÈLE. - Dans cette partie nous traitons de la mise en équation du problème de séchage d'un matériau diphasique en situation de retrait idéal [19].

Le retrait étant supposé idéal et compte tenu des diverses fonctions que nous venons de passer en revue, le couplage entre le comportement mécanique du matériau et le séchage est exprimé de façon explicite par une relation expérimentale donnant le retrait volumique $\left(R_{\mathrm{v}}\right)$ ainsi que la vitesse de retrait $\left(\mathrm{d} R_{\mathrm{v}} / \mathrm{d} t\right)$ en fonction de la teneur en eau mo enne du matériau. Le choix d'un modèle diffusif, pour décrire le séchage de ces matériaux diphasiques, est tout à fait en accord avec les travaux de Puiggali et al. [28] qui ont analysé ce type de choix en fonction des dimensions caractéristiques des espaces occupés par le fluide, des dimensions de la pièce à sécher et des conditions de séchage. Cependant pour intégrer les spécificités du matériau déformable nous mettons alors en place un modèle diffusif avec des conditions à la limite du type frontière libre. Le déplacement de la frontière est évidemment déduit de la fonction retrait. Ce modèle est écrit dans un repère fixe centré au point de symétrie de la géométrie étudiée (Fig. 2.3).

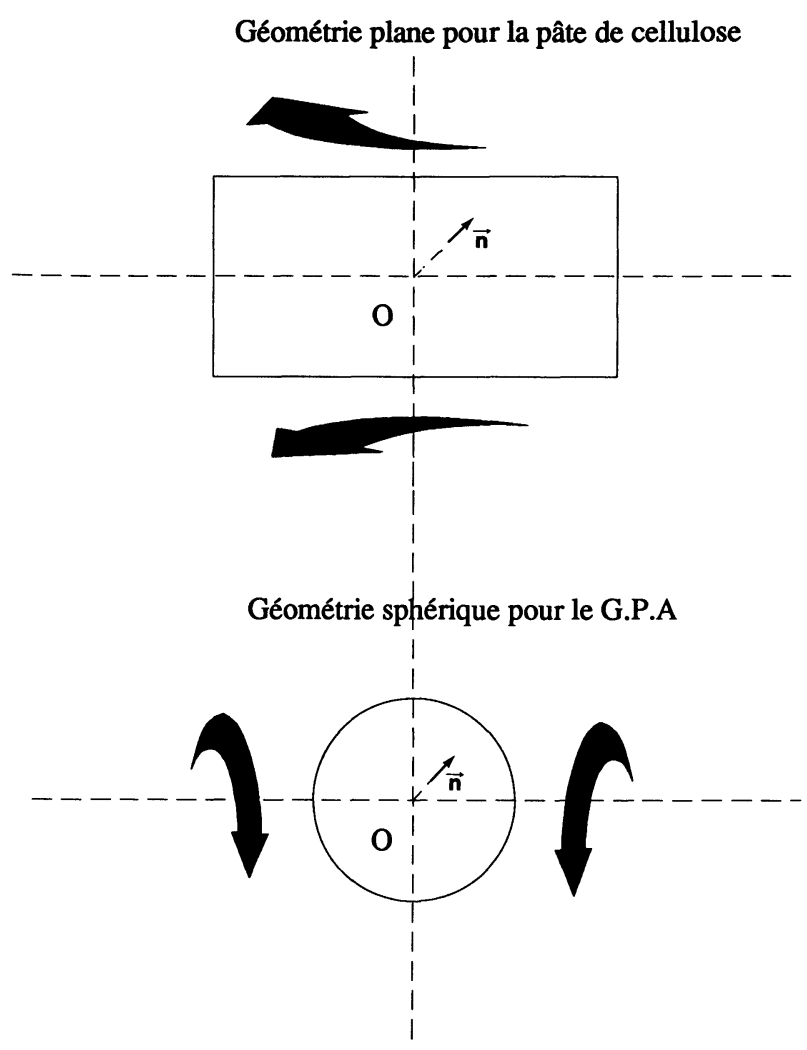

Fig. 2.3. - Géométries des matériaux.

[Geometries of materials.]

Bilan de masse

$$
\begin{array}{ll}
\text { Phase solide } & \frac{\partial \rho_{\mathrm{s}}^{\mathrm{a}}}{\partial t}+\operatorname{div}\left(\rho_{\mathrm{s}}^{\mathrm{a}} \boldsymbol{\mho}_{\mathrm{s}}\right)=0 \\
\text { Phase liquide } & \frac{\partial \rho_{1}}{\partial t}+\operatorname{div}\left(\rho_{1} \boldsymbol{\mho}_{1}\right)=0
\end{array}
$$

dans ces équations $t$ est le temps, $\boldsymbol{\vartheta}_{1}$ et $\boldsymbol{\vartheta}_{\mathrm{s}}$ sont respectivement les vitesses de déplacement de la phase liquide (eau) et de la phase solide.

Bilan d'énergie. - Nous nous plaçons dans l'hypothèse de l'équilibre thermique local, on aura

$$
\left(\rho C_{\mathrm{p}}\right) \frac{\partial T}{\partial t}+\operatorname{div}\left(\mathbf{J}_{\mathrm{e}}\right)=0
$$

Dans cette relation $\left(\rho C_{\mathrm{p}}\right)$ est la chaleur massique du matériau humide, $T$ la température de ce même matériau et $\mathbf{J}_{\mathrm{e}}$ la densité du flux d'énergie exprimée comme :

$$
\mathbf{J}_{\mathrm{e}}=-\lambda \operatorname{grad} T
$$

avec $\lambda$ la conductivité thermique du matériau humide. 
Ce bilan d'énergie est écrit de façon simplifiée, nous n'avons pas pris en compte la déformation du matériau, son effet sur l'équation de l'énergie ferait apparaître un terme convectif.

Expression du bilan de masse en terme de teneur en $e a u$. - La teneur en eau base sèche $W$ peut s'écrire sous la forme suivante :

$$
W=\frac{\rho_{1}}{\rho_{\mathrm{s}}^{\mathrm{a}}}
$$

Le flux diffusif de la phase fluide par rapport à la phase solide $\left(J_{m}^{s}\right)$ est défini comme suit :

$$
\mathbf{J}_{\mathrm{m}}^{\mathrm{s}}=\rho_{1}\left(\boldsymbol{\mho}_{1}-\boldsymbol{\mho}_{\mathrm{s}}\right)
$$

Le choix d'un modèle du type diffusif nous permet d'écrire $\mathbf{J}_{\mathrm{m}}^{\mathrm{s}}$ de la manière suivante :

$$
\mathbf{J}_{\mathrm{m}}^{\mathrm{s}}=-D_{\mathrm{m}} \operatorname{grad}\left(\rho_{1}\right)
$$

où $D_{\mathrm{m}}$ est le coefficient de diffusion de masse que l'on précise dans le paragraphe suivant.

La masse du composant anhydre est constante, et, de plus, en supposant que ce composant est uniformément réparti, le gradient de $\rho_{\mathrm{s}}^{\mathrm{a}}$ est alors nul. Cette hypothèse suggérée par Crapiste et al. [13] permet d'évaluer analytiquement la vitesse de la phase solide. En ce qui concerne l'évaluation des champs de teneurs en eau cette hypothèse, en toute rigueur fausse, n'est pas retenue. S'affranchir complètement de cette hypothèse nécessite une résolution numérique de l'équation de continuité de la phase solide, démarche qui n'a pas été utilisée dans ce travail.

Nous avons donc :

$$
\rho_{\mathrm{s}, t=0}^{\mathrm{a}} V(t=0)=\rho_{\mathrm{s}}^{\mathrm{a}} V(t)
$$

Cette relation peut se mettre sous la forme :

$$
\frac{\rho_{\mathrm{s}, t=0}^{\mathrm{a}}}{\rho_{\mathrm{s}}^{\mathrm{a}}}=R_{\mathrm{v}}
$$

Pour nos matériaux et nos géométries, le retrait est isotrope, nous nous ramenons à un déplacement monodimensionnel. Dans ce cas la vitesse de déplacement de la phase solide, $\boldsymbol{\vartheta}_{\mathrm{s}}$, est portée par le vecteur unitaire n (Fig. 2.3) et peut être calculée à partir de l'équation de conservation de la phase solide et de la fonction retrait [13] :

$$
\boldsymbol{\vartheta}_{\mathrm{s}}(x, t)=\frac{x}{3 R_{\mathrm{v}}(\langle W\rangle)} \frac{\mathrm{d} R_{\mathrm{v}}(\langle W\rangle)}{\mathrm{d} t} \mathbf{n}
$$

où $\langle W\rangle$ représente la teneur en eau moyenne du matériau à l'instant $t$ considéré, teneur en eau définie comme :

$$
\langle W\rangle=\frac{1}{V(t)} \int_{V(t)} W \mathrm{~d} V
$$

Le modèle de séchage ainsi établi est alors défini par les équations suivantes:

Bilan de masse

$$
\begin{aligned}
\rho_{\mathrm{s}}^{\mathrm{a}}\left(\frac{\partial W}{\partial t}+\boldsymbol{\vartheta}_{\mathrm{s}} \cdot \operatorname{grad}(W)\right)= \\
=\operatorname{div}\left(\rho_{\mathrm{s}}^{\mathrm{a}} D_{\mathrm{m}} \operatorname{grad}(W)\right)
\end{aligned}
$$

Bilan d'énergie

$$
\left(\rho C_{\mathrm{p}}\right) \frac{\partial T}{\partial t}=\operatorname{div}(\lambda \operatorname{grad}(T))
$$

Ce modèle s'accompagne de relations complémentaires qui sont les équations (1.1), (2.14) et (2.15) et est précisé par les conditions aux limites et initiales correspondant aux problèmes illustrés par la figure 2.3.

Conditions aux limites. - Les conditions à la limite sont écrites respectivement sur la surface d'échange $(\Gamma)$, mobile au cours du temps, et au centre de symétrie de l'élément ( 0$)$.

Flux de masse

$-\left.\rho_{\mathrm{s}}^{\mathrm{a}} D_{\mathrm{m}} \operatorname{grad}(W) \cdot \mathbf{n}\right|_{x=x(\Gamma)}=h_{\mathrm{c}}\left(C_{\Gamma}-C_{\infty}\right)$

$$
\left.\operatorname{grad}(W) \cdot \mathbf{n}\right|_{x=0}=0
$$

Flux de chaleur

$$
\begin{gathered}
-\left.\lambda \operatorname{grad}(T) \cdot \mathbf{n}\right|_{x=x(\Gamma)}=h_{\mathrm{T}}\left(T_{\Gamma}-T_{\infty}\right)+ \\
+\Delta H_{\mathrm{v}} h_{\mathrm{c}}\left(C_{\Gamma}-C_{\infty}\right) \\
\left.\operatorname{grad}(T) \cdot \mathbf{n}\right|_{x=0}=0
\end{gathered}
$$

Dans ces équations, $h_{\mathrm{c}}$ et $h_{\mathrm{T}}$ sont respectivement les coefficients d'échange de masse et de chaleur et sont déduits de la théorie de la couche limite [29] ou identifiés à partir des cinétiques de séchage en régime isenthalpe, $\Delta H_{\mathrm{v}}$ est l'enthalpie de vaporisation de l'eau à la température $T_{\Gamma}, C_{\Gamma}$ est la concentration de vapeur d'eau à la surface du matériau et $C_{\infty}$ celle dans l'air de séchage. $C_{\Gamma}$ est égale à la concentration à saturation pour $T_{\Gamma}$ en régime isenthalpe puis égale à celle donnée par l'équilibre hygroscopique, lorsque la surface du matériau n'est plus saturée (Figs. 1.1a et b).

Conditions initiales. - A l'instant $t=0$ les champs de teneur en eau et de température sont uniformes dans le matériau humide

$$
\begin{aligned}
& \left.W(x)\right|_{t=0}=W_{0} \\
& \left.T(x)\right|_{t=0}=T_{0}
\end{aligned}
$$

2.3 LE COEFFICIENT DE TRANSPORT DE MASSE.. Le coefficient de transport de masse peut être identifié des deux manières suivantes : 
i) Tanaka [30] propose d'utiliser l'équation de déplacement écrite pour un champ de déplacement unidirectionnel $\mathcal{U}(x, t)$ :

$$
\frac{\partial \mathcal{U}}{\partial t}=\mathcal{A} \nabla^{2} \mathcal{U}
$$

avec les conditions à la limite suivantes :

$$
\begin{aligned}
& \left.\mathcal{U}\right|_{x=\text { centre }}=0 \\
& \left.\sigma_{\mathrm{rr}}\right|_{x \rightarrow x_{\text {eq }}}=0 .
\end{aligned}
$$

ii) Pour sa part, Crank [31], a utilisé l'équation de diffusion suivante :

$$
\frac{\partial W}{\partial t}=\mathfrak{D} \nabla^{2} W
$$

avec les conditions à la limite suivantes :

$$
\begin{gathered}
\left.\frac{\partial W}{\partial x}\right|_{x=\text { centre }}=0 \\
\left.W\right|_{x=\text { surface }}=W_{\text {éq }}
\end{gathered}
$$

Ces choix étant faits, on accède aux coefficients de transport de masse isothermes en résolvant analytiquement à coefficient constant l'une ou l'autre des équations ci-dessus. Les deux équations ont des solutions analogues dont on rappelle ici la forme :

Cas de la géométrie sphérique

$$
\frac{X-X_{\text {eq }}}{X_{0}-X_{\text {eq }}}=\frac{6}{\pi^{2}} \sum_{n=1}^{n=\infty} \frac{1}{n^{2}} \exp \left[-n^{2} \pi^{2} \frac{D_{\mathrm{m}} t}{\ell^{2}}\right]
$$

Cas de la plaque

$$
\begin{aligned}
\frac{X-X_{\text {éq }}}{X_{0}-X_{\text {é }}}= & \frac{8}{\pi^{2}} \sum_{n=1}^{n=\infty} \frac{1}{(2 n+1)^{2}} \times \\
& \times \exp \left[-\frac{(2 n+1)}{4} \pi^{2} \frac{D_{\mathrm{m}} t}{\ell^{2}}\right]
\end{aligned}
$$

dans ces relations $X$ est la valeur moyenne de l'une des variables $\mathcal{u}$ ou $W$.

Ces deux techniques sont tout à fait analogues pour identifer le coefficient de transport de masse. Ce coefficient apparaît donc comme intrinsèque au matériau étudié.

Il est alors légitime d'identifier le coefficient de diffusion $\left(D_{\mathrm{m}}\right)$ de l'équation de bilan de masse à ce coefficient de transport. Les valeurs trouvées pour $D_{\mathrm{m}}$ sont de l'ordre de $10^{-8}\left(\mathrm{~m}^{2} / \mathrm{s}\right)$ pour la pâte de cellulose et de l'ordre de $10^{-10}\left(\mathrm{~m}^{2} / \mathrm{s}\right)$ pour le G.P.A., ces valeurs confirment le choix d'une diffusion Fickienne (Crank, [31], p. 262).

Une fois le modèle établi et les principaux coefficients identifiés nous nous proposons dans le paragraphe suivant de valider la modélisation globale par des comparaisons avec quelques expériences de séchage. Cette validation faite, l'influence de la vitesse de retrait sur l'équation en teneur en eau est analysée.

\section{Simulation du séchage.}

3.1 RÉSOLUTION NUMÉRIQUE. - Le modèle décrit ci-dessus a été résolu à l'aide d'une méthode aux éléments finis, la grille du maillage suit à tout instant la déformation du domaine physique considéré. Nous reportons ici les étapes essentielles de la résolution numérique ainsi que les choix effectués :

- la discrétisation spatiale est en éléments finis avec interpolation linéaire,

- la grille du maillage suit le mouvement de la phase solide,

- la discrétisation temporelle se fait suivant le schéma d'Euler,

- les systèmes linéaires découlant de cette discrétisation sont résolus à l'aide de la méthode de Choleski.

3.2 VAlidation DU MODÈle. - Sur les figures 3.1a et $3.1 \mathrm{~b}$ sont reportées une comparaison entre une cinétique expérimentale et une obtenue avec le modèle numérique pour nos deux matériaux sous des contraintes de séchage indiquées sur les figures. On notera la bonne concordance générale entre l'expérience et le modèle. Il ne nous a pas été possible de mesurer des profils de teneur en eau, aussi sur les figures $3.2 \mathrm{a}$ et $3.2 \mathrm{~b}$ ne sont reportés que les résultats des simulations, la présentation retenue permet aussi de suivre l'évolution de la dimension caractéristique (rayon de la bille, demi-épaisseur de la plaque).

3.3 INFLUENCE DU TERME CONVECTIF DANS L'ÉQUATION DE CONSERVATION. - Dans la mise en place du modèle (paragraphe 2) nous avons vu que la prise en compte du phénomène de retrait implique l'introduction d'un terme convectif $\left(\boldsymbol{\vartheta}_{\mathrm{s}} \cdot \operatorname{grad} W\right)$ dans l'équation de conservation de masse ; ce terme explicitant le transport du solvant lié au retrait.

Nous définissons les nombres de Biot de masse et Fourier de masse comme suit :

$$
\mathrm{Bi}=\frac{h_{\mathrm{c}} \ell_{0}}{D} \quad \text { Fo }=\frac{D_{\mathrm{m}} t}{2}
$$

Dans ces relations $D_{\mathrm{m}}$ est identifié comme suggéré au paragraphe $2.3, h_{\mathrm{c}}$ est le coefficient de transport de masse à l'interface entre l'air de séchage et la surface du matériau, $\ell_{0}$ est la dimension caractéristique du matériau à l'instant initial et $\ell(t)$ sa dimension instantanée. On notera ici que le nombre de Biot dans le cas parfaitement 1D (géométrie plane) est bien relié au flux de masse échangé avec l'air extérieur. Dans le cas radial (géométrie sphérique) il faudrait tenir compte dans la définition de l'évolution de la surface d'échange au cours du retrait. Nous 

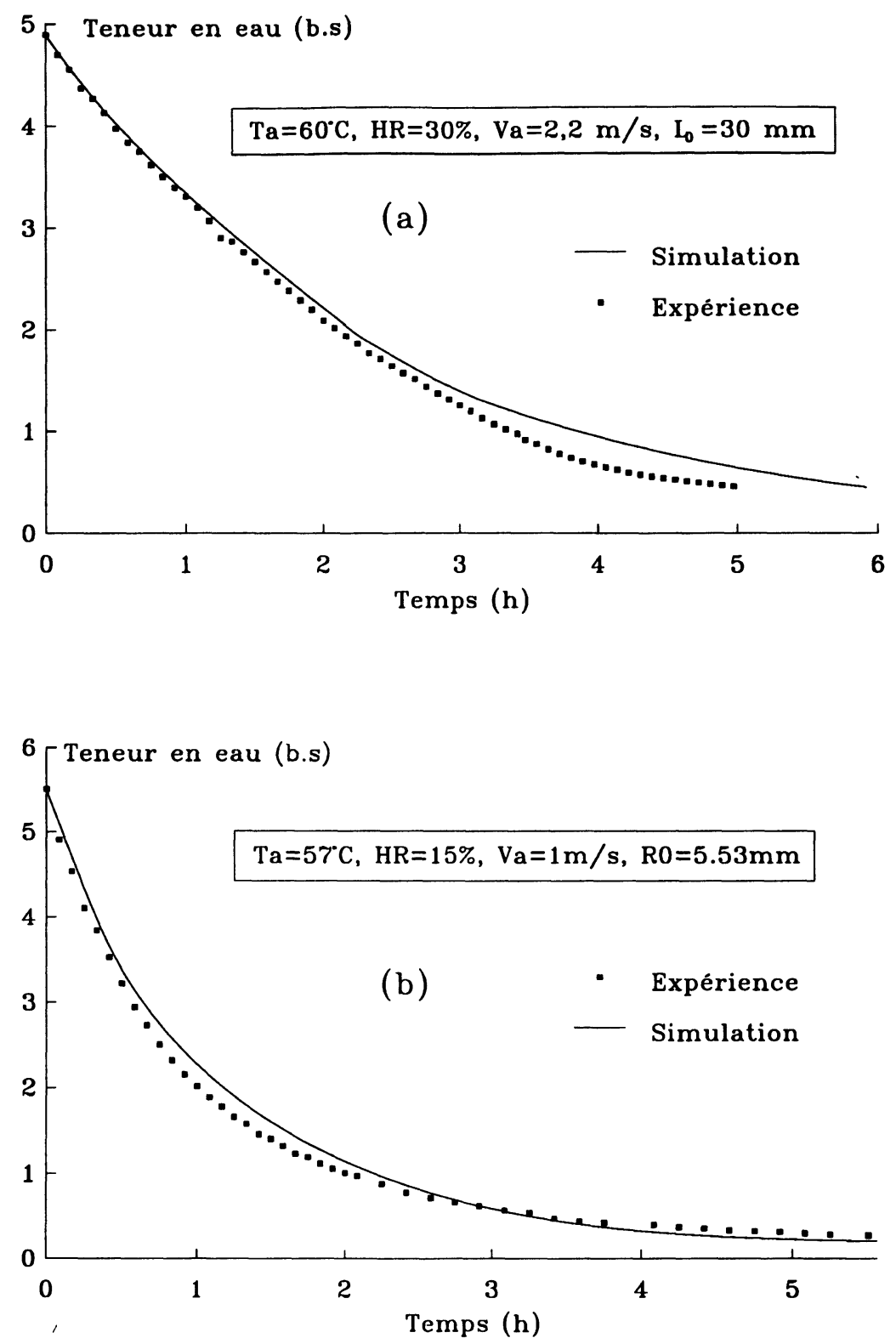

Fig. 3.1. - Cinétiques de séchage : a) cellulose purifiée, b) G.P.A.

[Drying kinetics : a) regenerated cellulose, b) polyacralamid gel (G.P.A.).]

n'avons pas retenu une définition plus complexe et moins maniable.

Nous nous proposons dans ce paragraphe de montrer l'influence de la prise en compte du terme $\boldsymbol{\vartheta}_{\mathrm{s}} \cdot \operatorname{grad}(W)$ dans le transport de masse et donc d'analyser le domaine de validité d'un modèle classique de séchage pour prendre en compte la spécificité du matériau déformable. Pour ce faire nous avons calculé des cinétiques pour divers nombres de Biot correspondants aux gammes suggérées par nos matériaux, avec ou sans le terme $\boldsymbol{\vartheta}_{\mathrm{s}} \cdot \operatorname{grad}(W)$, cependant dans les deux cas le retrait est maintenu et est caractérisé par les fonctions propres aux matériaux.

Les résultats reportés sur les figures $3.3 \mathrm{a}$ et $3.3 \mathrm{~b}$ montrent que quel que soit le matériau l'importance $\mathrm{du}$ terme convectif croît avec le nombre de Biot; ceci correspond à l'apparition de gradients de teneur en eau de plus en plus importants.

Pour nos matériaux et les types d'essais de séchage que nous avons réalisés les nombres de Biot sont respectivement autour de $2 \times 10^{3}$, pour la plaque de cellulose, et de $2 \times 10^{6}$, pour la bille de G.P.A. Il était donc nécessaire de modifier le modèle diffusif.

Pour s'en convaincre, regardons les graphes des figures $3.4 \mathrm{a}$ et $3.4 \mathrm{~b}$. Il s'agit d'une représentation dans un espace Biot-Fourier des iso-écarts entre la solution du modèle complet et celle du modèle réduit (purement diffusif). 

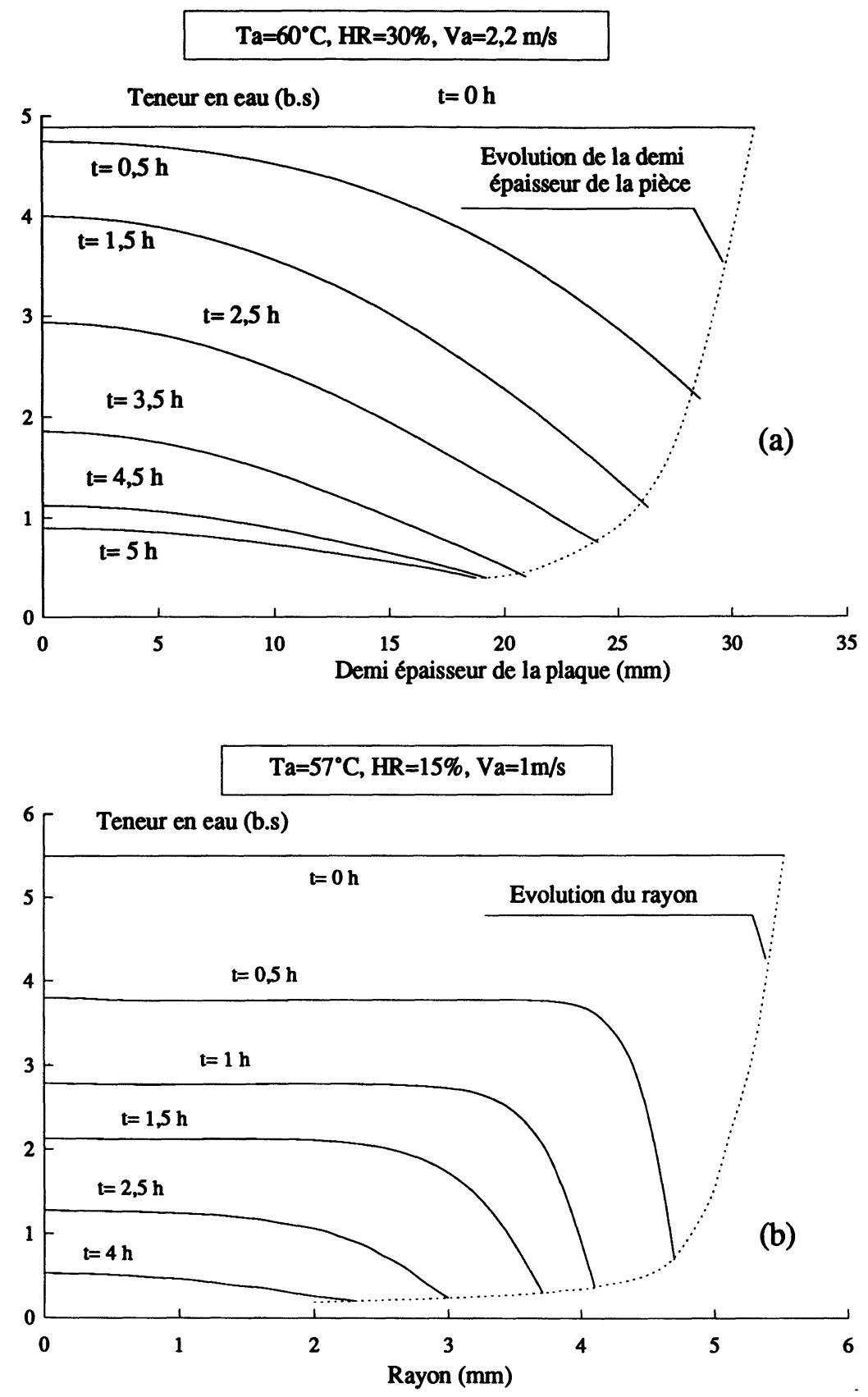

Fig. 3.2. - Profils de teneurs en eau pour différents instants : a) cellulose purifiée, b) G.P.A.

[Internal moisture fields at different times : a) regenerated cellulose, b) polyacralamid gel (G.P.A.).]

Les iso-écarts sont définis comme :

$$
E_{w}=\left[W_{\text {avec vitesse }}-W_{\text {sans vitesse }}\right]_{\mathrm{Fo}=\mathrm{Cte}}
$$

Dans cette représentation et compte tenu des hypothèses retenues $\left(D_{\mathrm{m}}\right.$ et $h_{\mathrm{c}}$ constants) le suivi d'une cinétique de séchage se fait sur une horizontale $(\mathrm{Bi}=\mathrm{Cte})$.

Sur ces mêmes graphes nous avons reporté les isoteneurs en eau solutions du problème complet en partant d'un matériau saturé.
Ces graphes confirment la croissance de l'écart avec le nombre de Biot et font apparaître deux zones, séparées par une bifurcation : une première à faible Biot où l'écart reste relativement faible et une seconde à Biot plus élevé où l'écart croît notablement. Ainsi c'est seulement dans la première zone que l'on pourrait négliger $\boldsymbol{\mho}_{\mathrm{s}} \cdot \operatorname{grad}(W)$ dans l'équation de transport.

Pour pousser plus loin l'analyse du paragraphe précédent, nous avons abordé l'influence du module 

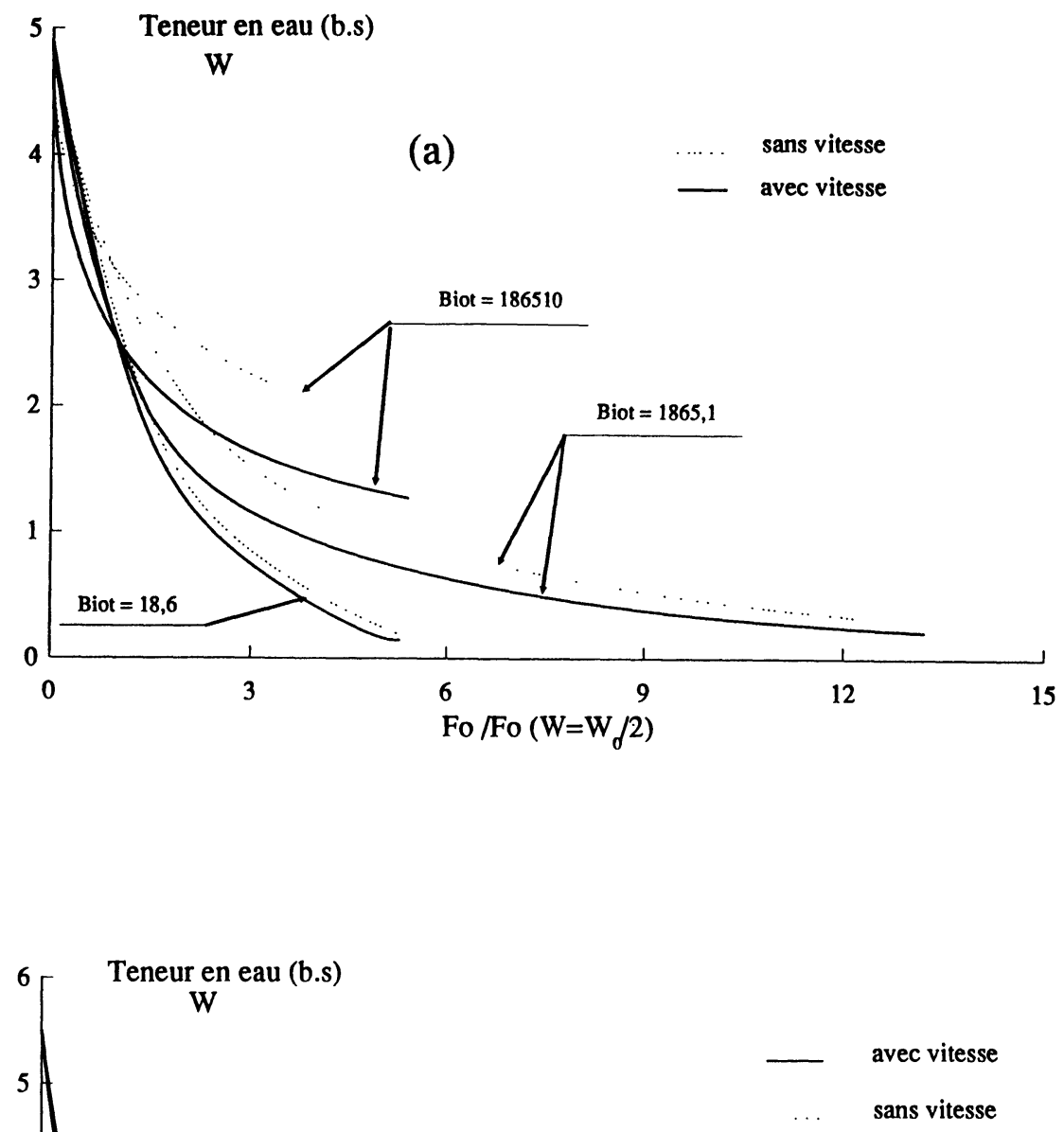

(b)

Fig. 3.3. - Cinétiques de séchage à Biot donné avec ou sans prise en compte du terme convectif : a) cellulose purifiée, b) G.P.A.

[Comparison between drying kinetics taking the convective term into account or not, at fixed Biot number: a) regenerated cellulose, b) polyacralamid gel (G.P.A.).]

du retrait volumique $\left(V_{0} / V_{\text {sec }}\right)$ sur le transport de masse. Il s'agit d'une généralisation par la fonction retrait à d'autres matériaux que ceux qui ont supporté ce travail, la seule restriction restante est la condition de retrait idéal. Les résultats reportés sur la figure 3.5 présentent l'écart pour différentes fonctions retrait. Ces résultats montrent clairement que plus le retrait est important, plus l'influence du terme convectif est marquée. Ainsi un retrait faible ne nécessite pas une analyse complémentaire en nombre de Biot, alors qu'à l'inverse un retrait important oblige à examiner le nombre de Biot pour pouvoir décider du modèle à retenir. 

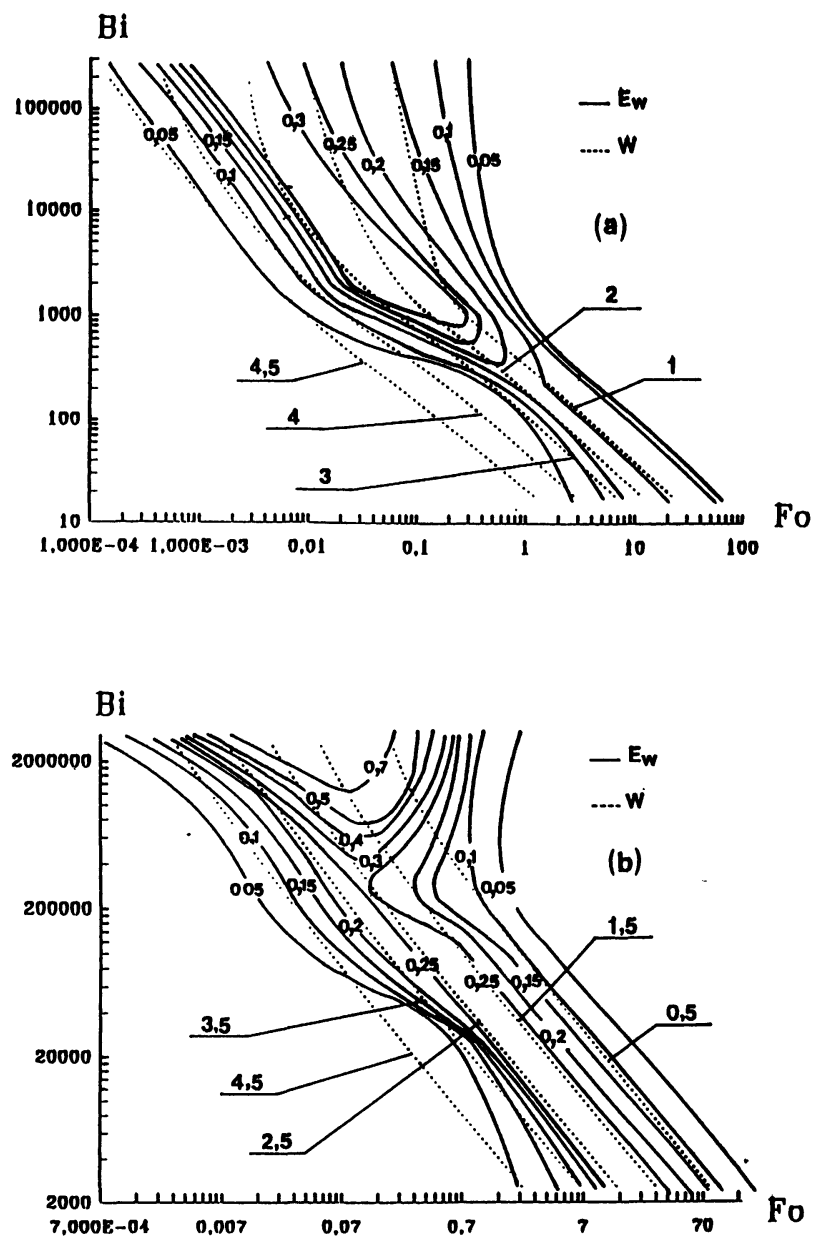

Fig. 3.4. - Iso-différences $\left(E_{\mathrm{w}}\right)$ : a) cellulose purifiée, b) G.P.A.

[Iso-deviations $\left(E_{\mathrm{w}}\right)$ : a) regenerated cellulose, b) polyacralamid gel (G.P.A.).]

\section{Conclusion.}

Dans ce texte, partant de deux cas concrets de séchage de matériaux déformables, nous avons analysé les diverses propriétés nécessaires à une bonne compréhension puis à la modélisation des processus.

L'analyse en terme de retrait nous a amené à développer un modèle de transport de masse coulant diffusion et dé lacement mo ennant les

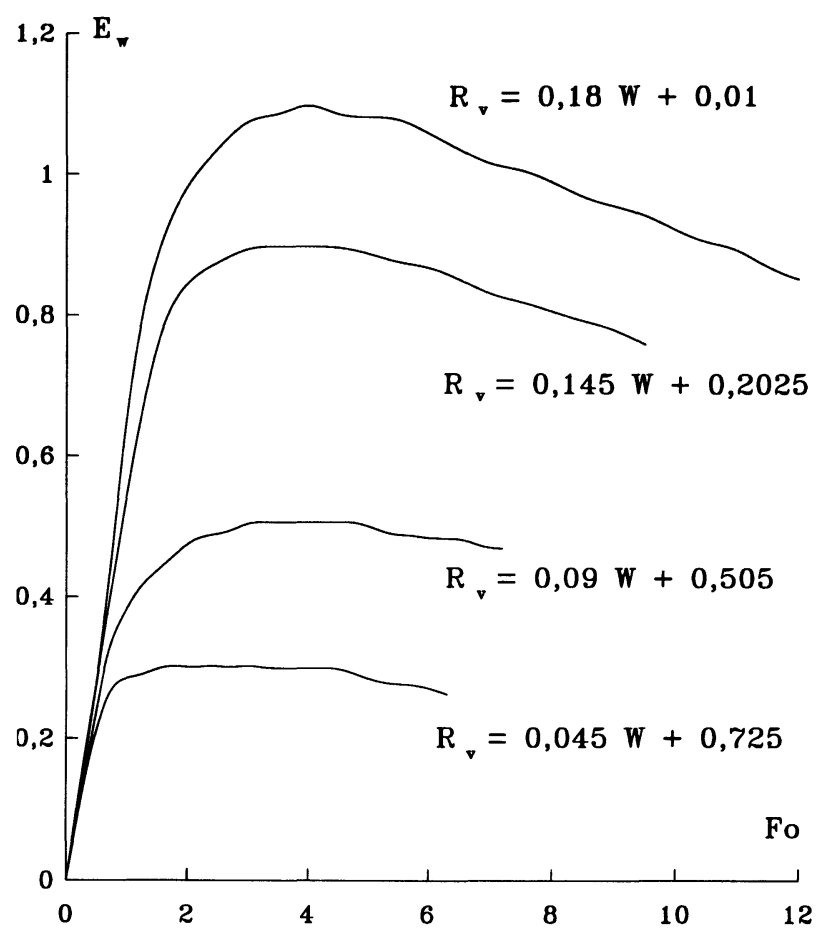

Fig. 3.5. - Evolution de l'écart $E_{\mathrm{w}}$ en fonction du nombre de Fourier pour différents modules de retrait.

[Deviation $\left(E_{\mathrm{w}}\right)$ as a function of the Fourier number for different shrikage modulus.]

hypothèses au demeurant drastiques, que nous rappelons ici :

- retrait idéal,

- matériau diphasique.

L'équation de l'énergie a été traitée de façon simplifiée. Nous avons par ailleurs limité notre étude à des déplacements monodimensionnels, ce qui nous permet d'expliciter la vitesse de déplacement du solide à partir de l'équation de conservation de la masse de la phase solide.

Une attention toute particulière a été portée au terme $\boldsymbol{\vartheta}_{\mathrm{s}} \cdot \operatorname{grad}(W)$ afin de préciser dans quelles situations (nombre de Biot et vitesse de la phase solide) les modèles classiques de séchage purement diffusifs devaient évoluer vers des modèles du type 


\begin{tabular}{|c|c|}
\hline Pâte de cellulose & Gel du Polyacrilamide \\
\hline \multicolumn{2}{|c|}{ Masse volumique $\left(\mathrm{kg} / \mathrm{m}^{3}\right)$} \\
\hline $\begin{array}{l}\rho(W)=A \cdot \exp \left(B \cdot W+C \cdot W^{2}\right)+E \cdot W+G \\
A=623 \\
B=-0,09 \\
C=-1,07 \\
E=39,4 \\
G=824,9\end{array}$ & $\begin{array}{l}\rho(W)=\rho_{1}+\frac{A}{1+W} \\
\rho_{1}=1000 \\
A=346\end{array}$ \\
\hline \multicolumn{2}{|c|}{ Chaleur massique $\left(\mathrm{J} / \mathrm{kg} /{ }^{*}\right)$} \\
\hline $\begin{array}{l}C_{\mathrm{p}}(W, T)=\frac{C_{\mathrm{pc}}+C_{\mathrm{pe}} \cdot W}{1+W} \\
C_{\mathrm{pc}}=1112+4,85 \cdot(T-273) \\
C_{\mathrm{pe}}=A \cdot T^{3}+B \cdot T^{2}+C \cdot T+D \\
A=0,75 \times 10^{-4} \\
B=-0,944 \times 10^{-2} \\
C=0,449 \\
D=4186,74\end{array}$ & $\begin{array}{l}C_{\mathrm{p}}(W, T)=\frac{C_{\mathrm{pg}}+C_{\mathrm{pe}} \cdot W}{1+W} \\
C_{\mathrm{pc}}=1660 \\
C_{\mathrm{pe}}=A \cdot T^{3}+B \cdot T^{2}+C \cdot T+D \\
A=0,75 \times 10^{-4} \\
B=-0,944 \times 10^{-2} \\
C=0,449 \\
D=4186,74\end{array}$ \\
\hline \multicolumn{2}{|c|}{ Diffusivité thermique $\left(\mathrm{m}^{2} / \mathrm{s}\right)$} \\
\hline$a=\frac{0,45+0,04 W}{\rho(W) \cdot C_{\mathrm{p}}(W, T)}$ & $a=2 \times 10^{-7}$ \\
\hline \multicolumn{2}{|c|}{ Retrait volumique } \\
\hline$R_{\mathrm{v}}=1440 \cdot(1+W) / \rho(W)$ & $R_{\mathrm{v}}=0,18 W+0,01$ \\
\hline \multicolumn{2}{|c|}{ Coefficient de diffusion de masse $\left(\mathrm{m}^{2} / \mathrm{s}\right)$} \\
\hline$D_{\mathrm{m}}=2 \times 10^{-8}$ & $D_{\mathrm{m}}=9 \times 10^{-10}$ \\
\hline
\end{tabular}




\section{Bibliographie}

[1] Mujumdar A. S., Handbook of industrial drying (Marcel Dekker Inc., New York) 1987.

[2] Nadeau J. P., Puiggali J. R., De l'analyse d'un produit à la conception d'un séchoir, Entropie 149 (1989) 41-47.

[3] Aregba W., JomaA W., Nadeau J. P., Puiggal J. R., Deux procédés électriques de séchage: infrarouge et micro-onde, Collection « Récents progrès en génie des procédés " (Lavoisier Technique et Documentation, Paris) vol. 3 (1989) 240-245.

[4] Berger D., Pei D. C. T., Drying of hygroscopic capillary porous solids, a theoritical approach, Int. J. Heat Mass Transfer 16 (1973) 293-302.

[5] Bories S., Recent advances in modelisation of coupled heat and mass transfer in capillaryporous bodies, Proc. IDS'88 1 (1988) 47-61.

[6] ILIC M., TURNER I. W., Drying of a wet porous material, Appl. Math. Modelling 10 (1986) 1624.

[7] Quintard M., Puiggali J. R., Modèles de séchage de produits capillaro-poreux, propriétés, applications, Collection « Récents progrès en génie des procédés » (Lavoisier technique et Documentation, Paris) vol. 1 (1987) 272-277.

[8] Harmathy T. Z., Simultaneous moisture and heat transfer in porous systems with particular reference to drying, IGEC Fundamentals 8 (1969) 92-103.

[9] Stanish M. A., Schajer G. S., Kayihan F., A mathematical modeling of drying for hygroscopic porous media, AIChE J. 32 (1986) 1301-1311.

[10] WhitAKer S., Heat and mass transfer in granular porous media : a theory of drying, Advances in Drying, Hemisphere Publishing Corporation Ed., New York) vol. 1 (1980) 23-61.

[11] Plumb O. A., Spolek G. A., Olmstead B. A., Heat and mass transfer in wood during drying, Int. J. Heat Mass. Transfer 28 (1985) 1669-1678.

[12] Puiggali J. R., Quintard M., Properties and simplifying assumptions for classical drying models ; Advances in Drying (Hemisphere Publishing Corporation, New York) vol. 5 (1990).

[13] Crapiste G. H., Rostein E., Whitaker S., Drying cellular material. I : Mass transfer theory, Chem. Eng. Sci. 43 (1988) 2919-2928 ; II : Experimental and numerical results, Chem. Eng. Sci.

[14] Rao V. N. M., HamanN D. D., Hammerle J. R., Stress analysis of a viscoelastic sphere subjected to temperature and moisture gradients, J. Agricultural Eng. Res. 20 (1975) 283-293.

[15] Haghighi K., Segerlind L. J., Failure of biomaterials subjected to temperature and moisture gradients using the finite element mehod : I -
Thermo-hydro viscoelasticity, Trans. ASAE 31 (1988) 930-937.

[16] Sir G. C., Michopoulos J. G., Chou S. C., Hyrothermoelasticity (Martinus Nijhoff Publishers, Dordrecht) 1986.

[17] Kechaou N., Roques M., Séchage convectif de billes de Polyacrylamide, Modélisation des transferts internes et externes, Actes de la rencontre S.F.T. "Thermique et génie des procédés", (Nancy, France) tome 1 (1989) 239-250

[18] Collard J. M., Arnaud G., Fohr J. P., Transfert d'humidité et déformation d'une plaque d'argile pendant le séchage, Actes de la rencontre S.F.T. "Thermique et génie des procédés ", tome 1 , Nancy (1989) 239-250.

[19] Aregba W., Séchage d'un gel et d'une pâte. Processus internes et procédés adaptés, Thèse de l'Université de Bordeaux (1989) p. 1.

[20] Geissler E., Hecht A. M., Gel deswelling under reverse osmosis II, J. Chem. Phys. 77 pp. 1548 1553.

[21] TANAKA T., Les gels, pour la Science 41 (1981) 62-78.

[22] Baquey Ch., Chauveaux D., Barbie Ch., Poustis J., Pommier J. C., Suitability of regenerated cellulose as a sealant to fix hip prosthesis according to an original procedure. The third word biometerials congress (Kyoto, Japan) April 1988.

[23] Bizot H., Riou N., Multon J. L., Guide pratique pour la détermination des isothermes de sorption et de l'activité de l'eau, Sc. Aliments, $\mathrm{n}^{\circ}$ hors série (1987).

[24] Nelson R. M., A model for the sorption of water vapor by cellulosic materials, Wood Fiber Sci. 15 (1983) 8-22.

[25] D'ARCY R. L., WATt I. C., Analysis of sorption isotherms of non-homogeneous sorbents, Trans Faraday Soc. 66 (1970) 1236-1245.

[26] Kneule F., Le Séchage (Ed. Eyrolle, CNAM Paris) 1964.

[27] Auriat R., Benet J. C., Structure interne du caoutchouc naturel lors du séchage, C. R. Hebd. Acad. Sci. Paris Sér. II 309 (1989) 781-786.

[28] Puiggali J. R., Quintard M., Whitaker S., Drying granular porous media: Gravitational effects and the role of diffusion models, Drying Technol. 6 (1988) 601-629.

between a drying material and an external flow, Drying's 86, Ed. A. S. Mujumdar (Hemisphere Publishing Corporation, New York) 1 (1986) 105-112.

[30] TanaKa T., Fillmon D. J., Kinetics of swelling of gels, J. Chem. Phys. 70 (1979) 1214-1218.

[31] Crank J., The Mathematics of Diffusion, $2^{\mathrm{e}}$ édition (Clarendon Press, Oxford) 1975. 